\title{
Effects of a pain self-management intervention combining written and video elements on health-related quality of life among people with different levels of education
}

\author{
This article was published in the following Dove Press journal: \\ Journal of Pain Research \\ 20 August 2015 \\ Number of times this article has been viewed
}

\section{Carol Stalker \\ James Elander \\ Centre for Psychological Research, Department of Life Sciences, University of Derby, Derby, UK}

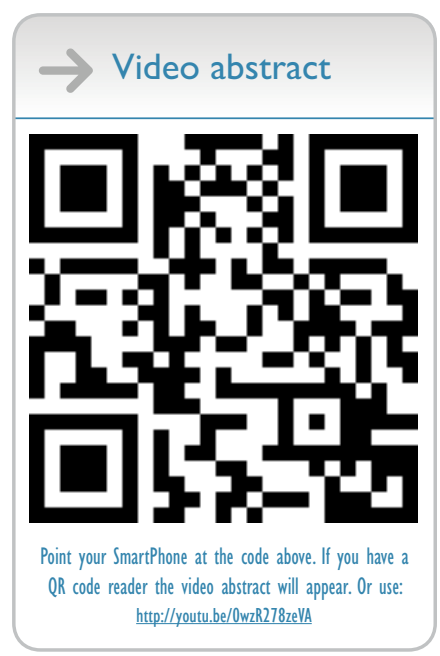

Correspondence: James Elander

Centre for Psychological Research, University of Derby, Kedleston Road, Derby DE22 IGB, UK

Tel +44I332593048

Email j.elander@derby.ac.uk

\begin{abstract}
Combining written and video material could increase the impact of health education for people with less education, but more evidence is needed about the impact of combined materials in different formats, especially in the context of chronic pain self-management. This study tested the impact of combining written information about self-managing chronic joint pain, which used language at a high reading level, with a DVD containing narrative video material presented directly by patients, using language at a lower reading level. Physical and mental health-related quality of life (36-Item Short Form Health Survey) was measured among 107 men with hemophilia before and 6 months after being randomly assigned to receive an information booklet alone or the booklet plus the DVD. Analysis of covariance was used to compare health outcomes between randomized groups at follow-up, using the baseline measures as covariates, with stratified analyses for groups with different levels of education. The DVD significantly improved mental health-related quality of life among those with only high school education. Video material could therefore supplement written information to increase its impact on groups with less education, and combined interventions of this type could help to achieve health benefits for disadvantaged groups who are most in need of intervention.
\end{abstract}

Keywords: DVD, hemophilia, video intervention

\section{Introduction}

Hemophilia is an inherited bleeding disorder in which blood clotting factors are missing or abnormal. The ankle and knee joints are especially susceptible to spontaneous bleeds, and repeated bleeding into joints causes hemophilic arthropathy, ${ }^{1}$ so chronic joint pain is a very common secondary symptom. ${ }^{2}$ One recent review concluded that "the majority of hemophilia patients will experience acute and/or chronic joint pain that is often debilitating and diminishes their quality of life", 3 and another concluded that the impact of hemophilia-related joint pain could be reduced by interventions to improve people's self-management of pain. ${ }^{4}$

Many health education interventions involve written material, which is usually high in information content but is often written at high reading levels, making it less accessible for people with less education or lower literacy. ${ }^{5,6}$ Video-based materials are usually more accessible in terms of language and communication, and are often developed as alternatives for hard-to-reach populations. ${ }^{7}$

Comparative studies offer little evidence that video has advantages over written material for people with less education or lower literacy, however. In a study of people's 
pain in the emergency department, a printed brochure and a video each reduced participants' self-rated pain. There were no differences in pain scores or ratings of how helpful the materials were, and the effects of the two types of material did not differ by educational level. ${ }^{8}$ A study of knowledge about colon cancer showed that written and video materials each improved knowledge by similar degrees and the effects did not differ by literacy level. ${ }^{9}$ A study of information recall about informed consent found there was a nonsignificant trend among participants with low reading comprehension for "enhanced" written materials (with simpler language, topic headings, and pictures) to be more effective than either the original written materials or video. ${ }^{10}$ In a study of knowledge of sleep apnea among people with limited literacy skills, video led to better scores in only two out of eleven areas of knowledge, compared with a simplified written brochure designed to be at an equivalent reading level. ${ }^{11}$

The relative advantages of written and video material may depend on the type of information and the way it is presented. Another study examined information recall among people with asthma, and showed that participants with lower levels of literacy who received video materials recalled more procedural information about using an inhaler, compared with those who received written materials. However, the video did not produce a similar comparative advantage in recall of factual information about asthma triggers. ${ }^{12}$ Narrative video, with information in the form of personal stories, has also been shown to be more effective than purely informational video, ${ }^{13,14}$ especially for people with less education. ${ }^{15}$

Written and video materials therefore have different strengths, so providing just video-based information would have limited benefits, whereas combined interventions have sometimes been more successful. A study of attitudes to blood donation among college students found that a written brochure and a video each had positive effects when delivered separately, but the brochure plus video produced larger improvements than either one alone. ${ }^{16}$ In a study of self-care behaviors among people with heart failure, those who received both written material and a DVD were more likely to increase their self-care behaviors than those who received only written materials, though they did not have better health outcomes. ${ }^{17}$ Among people with coronary artery disease, knowledge and health behaviors increased for those who received a printed booklet and those who received the booklet plus a video with patients explaining their own experiences. Among those who received both booklet and video there was a nonsignificant trend toward greater improvements in knowledge and health behaviors among patients with lower health literacy. ${ }^{18}$
Several of those studies matched the reading levels of the written and video material by producing written material at a very low reading level. ${ }^{10,11,18}$ However, reducing the reading level of written material does not necessarily increase its impact, ${ }^{11,19}$ whereas combining written and video material at different reading levels could optimize the benefits of each. A recent review concluded that more attention should be given to "message-equivalent" materials with similar content but presented in different formats, and that more explicit attention should be given during design to readability and patient input. ${ }^{20}$ Pairing written information at a relatively high reading level with more accessible video material would preserve the benefit of written material for factual information, and the video material would make that information more accessible for people with limited education or literacy, especially if the video involved patients and had narrative content. However, no studies to our knowledge have combined written and video materials that were designed to be at different reading levels.

In the present study, written information about selfmanaging hemophilia-related joint pain was supplemented with a DVD of patients presenting the same core messages in narrative video form. The written information had a relatively high reading level to optimize information content, whereas the DVD content used language at a lower reading level. The materials were specifically designed for the DVD to supplement the written material. We intended the DVD to enable more access to the written material by viewers with less education, so they could benefit to the same degree as participants with greater education. In this way, the advantages of the written material that were associated with higher reading levels could be retained, while the health education messages were made more available to those who might not have had access to them from the written information alone.

Most of the research on health education for people with lower health literacy has focused on people's understanding or recall of information related to health, but a review of interventions to improve health outcomes for people with low literacy recommended that more studies should include measures of actual health outcomes. ${ }^{21}$ Health-related quality of life, which includes physical functioning, mobility, everyday activities, social roles, and emotional wellbeing, is a health outcome that is strongly influenced by pain and pain management, ${ }^{22-24}$ and can be improved by pain-education programmes. $^{25}$

The present study was secondary to a randomized controlled trial of the booklet versus booklet plus DVD, in which the DVD reduced negative attitudes toward chronic 
pain self-management but did not improve health-related quality of life among the sample as a whole. ${ }^{26}$ In the present study, the impact of the DVD was tested separately among individuals with different levels of education, to assess the extent to which receiving the DVD improved quality of life specifically for those with less education. We intended the study findings to inform the development of health education interventions for disadvantaged groups with chronic pain, as well as the development of message-equivalent combined text and video interventions more generally.

\section{Methods}

\section{Participants and procedure}

The participants were members or registrants of the Haemophilia Society UK who had indicated willingness to take part in research coordinated by the Society. The inclusion criteria were having hemophilia A or B, age over 18 years, and good mailing status with the society, meaning the society had an up-to-date mailing address so the person could reasonably be expected to receive the materials necessary for participation. The exclusion criteria were medical conditions that complicate pain self-management, such as Alzheimer's disease. The sample size for the primary trial was designed to give 0.80 power to detect small-to-moderate effects, assuming $25 \%$ attrition over 6 months, which meant aiming for an initial baseline sample of 200 on the assumption that three-quarters would be successfully followed up at 6 months. ${ }^{26}$

Eligible individuals who gave informed consent and completed baseline assessment were randomized to receive an information booklet about self-managing hemophiliarelated joint pain, or the same information booklet plus a DVD containing a 25-minute video. Blind randomization was achieved by giving each participant a unique study number, then separating study numbers from all identifying information about participants, sorting them into a random sequence and then merging them with a computer-generated random sequence of $0 \mathrm{~s}$ and $1 \mathrm{~s}$, with equal numbers of $0 \mathrm{~s}$ and $1 \mathrm{~s}$ within each block of ten. Participants with study numbers associated with 0s were sent only the booklet and those with study numbers associated with $1 \mathrm{~s}$ were sent the booklet plus DVD.

Six months later, participants were sent a follow-up questionnaire and return envelope. After the 6-month follow-up, the DVD was sent to all those who previously received only the booklet. The research protocol was approved by the London Metropolitan University Research ethics committee.

\section{Measures}

Health-related quality of life was measured at both baseline and 6-months' follow-up using the RAND-36 (36-Item Short Form Health Survey), which gives scores on eight subscales, one of which is pain, and two summary scales, one for physical and one for mental health-related quality of life. ${ }^{27}$ Summary scales are computed as weighted aggregates of standardized subscale scores, and are then transformed to T-scores. ${ }^{28}$ These measures have good psychometrics and are frequently used in hemophilia research. ${ }^{29}$ The mental and physical summary scales were the primary outcome measures, and the pain subscale was also examined as a secondary outcome measure.

At 6 months, participants also rated how often they had used the materials and how helpful they found them, with participants in the booklet-plus-DVD group making separate ratings for the booklet and DVD. Participants in the booklet-only group were asked two questions: "how often did you read the booklet?" and "how helpful did you find the booklet?" Participants in the booklet-plusDVD group were asked those same two questions, plus two more questions about the DVD: "how often did you watch the DVD?" and "how helpful did you find the DVD?" The use ratings employed seven-point response scales ranging from "I haven't looked at it" to "I watched/read it more than once a week", and the helpfulness ratings employed four-point scales ranging from "not at all helpful" to "very helpful".

\section{Materials}

Both the booklet and DVD were designed as low-intensity interventions, to be mailed to participants with brief instructions and no need for personal instruction or clinical support.

People with hemophilia were closely involved in the development of both, consistent with recommended good practice for the development of health educational materials. ${ }^{20}$ Both booklet and DVD were designed to improve participants' readiness to self-manage chronic pain, and both addressed factors previously identified as influences on readiness to self-manage pain, including beliefs about costs and benefits, learning histories, contingencies, personal experience, modeling, verbal persuasion, and perceived barriers. ${ }^{30}$ In terms of Abraham and Michie's taxonomy of behavior change techniques, they provided information about behavior-health links and consequences; prompts to intention formation and barrier identification; prompts to goal-setting; general encouragement; and modeling behavior. ${ }^{31}$ 
However, the booklet placed greater emphasis on declarative informational content, whereas the DVD placed greater emphasis on attitudes and motivation. The booklet provided factual information about living with hemophilia and the benefits of greater pain self-management. It included information about types and causes of pain in hemophilia; the impact of pain on emotions and other aspects of life; positive and negative ways of using pain medications; and the benefits of active coping and exercise.

The DVD contained a 25-minute video covering the same topics as the booklet but with all the content delivered by five people with hemophilia. They presented narratives in their own words about their experiences of living and coping with joint pain, the impact of chronic joint pain on their lives, and ways they had adjusted life goals and values accordingly. All the content was delivered by the individuals who appeared in the film, who spoke directly to camera or whose spoken words accompanied video of them undertaking everyday tasks, leisure, and exercise inside and outside the home. Key messages were endorsed by two health professionals, a health psychologist and a physiotherapist, who also appeared in the film.

The content included descriptions of negative thinking, anger, and passive coping leading to low moods, restricted activities, and social isolation; participants' experiences of setting goals for everyday activities, social activities, and exercise; descriptions of the importance of keeping going with activities and setting personal goals; descriptions of the costs and benefits of using painkillers in negative and positive ways; and descriptions of the benefits of having hope and believing things could get better. The DVD could be watched in one continuous 25-minute viewing from beginning to end, or by selecting sections from an initial menu. Both booklet and DVD were designed to be used in as flexible a way as possible in order to maximize viewer engagement and allow participants to read or watch them in any context, including at home.

The specific intention was for the booklet to have a higher reading level than the DVD, in order to test the value of mismatched materials and the potential for video to help make written information more accessible for people with less education. Readability, estimated using the Online Utility Readability Calculator, ${ }^{32}$ was between grade levels 13 (Flesch-Kincaid) and 14 (SMOG) for the booklet, and between grade levels 10 (Flesch-Kincaid) and 11 (SMOG) for a transcription of DVD excerpts, indicating that, as intended, language in the DVD was less complex than in the booklet.
For the booklet, readability was around the level of higher education, because some of the concepts and information are necessarily complex, so that the booklet represents optimal presentation of information with few compromises or allowance made for readers with less education or literacy, whereas the DVD was intentionally aimed at individuals with less education or literacy. The readability of the booklet and the DVD transcript corresponded approximately to the levels of education of the groups of participants with most and least education. For participants with high school-only education, therefore, the booklet was above their expected reading level but the DVD used language closer to their expected reading level. For those with higher education, the booklet was at their expected reading level and the DVD used language below their expected reading level. Both booklet and DVD are available from the authors on request.

\section{Data analysis}

In preliminary analyses, differences between randomized groups and between those who did versus did not complete follow-up were tested with chi-squared tests (for level of education, hemophilia type and hemophilia severity), and $t$-tests (for age and baseline quality of life). Differences between education groups at baseline were tested with one-way analysis of variance (ANOVA), and changes in quality of life among the sample as a whole were tested with repeated-measures ANOVA.

In the main analyses, the effect of the DVD as a supplement to written information was tested with analysis of covariance (ANCOVA) in which follow-up quality of life scores were compared between randomized groups using baseline quality of life scores as covariates. This analysis tests for differences in outcome independently of any differences between groups at baseline, which can confound comparisons using scores representing changes from baseline to follow-up. ${ }^{33}$

There were two separate main analyses, one for each aspect of quality of life, with the specific baseline quality of life measure used as a covariate in each analysis, so that baseline physical quality of life was the covariate in the analysis of follow-up physical quality of life, and baseline mental quality of life was the covariate in the analysis of follow-up mental quality of life. In the secondary analysis of pain subscale scores, the same ANCOVA analyses were conducted, with follow-up pain subscale scores compared between randomized groups using baseline pain as the covariate. Effect sizes are reported as partial Eta squared $\left(\eta_{p}{ }^{2}\right)$, which describes the proportion of total variability 
in the dependent variable that is attributable to group differences.

The analyses were stratified by level of education, with separate analyses for participants with high school-only education, further education, and higher education. All randomized participants with follow-up data were included in the analyses, regardless of whether or not they reported reading and/or viewing the booklet and/or DVD, so the evaluation was not restricted to participants who had used the intervention materials as intended.

In supplementary analyses with chi-squared tests, participants' reported use of the materials and perceived helpfulness of the materials were compared between randomized groups and education groups to assess the extent to which any impact of the DVD on improved quality of life could be explained by differential use and perceived helpfulness. We also compared physical and mental quality of life, and pain subscale scores, between those who reported reading/ watching the booklet/DVD and those who did not, using $t$-tests, to assess the extent to which adherence contributed to the impact of the interventions.

\section{Results \\ Participants}

Of 209 individuals who were eligible, 191 were enrolled in the trial, of whom $131(69 \%)$ were followed-up 6 months later, and $107(56 \%)$ had complete education and quality of life data and comprised the study sample for the data analysis. Figure 1 shows participation in the form of a Consort flowchart. ${ }^{34}$

Among the 107 individuals in the study sample, ages ranged from 25 to 84 years with a mean of 50.51 years (standard deviation 12.39 years). All the participants were male. There were 85 (79\%) with hemophilia A and 18 (17\%) with hemophilia B (in four cases, hemophilia type was not known). There were $32(30 \%)$ with mild or moderate hemophilia and 73 (68\%) with severe hemophilia (in two cases, hemophilia severity was not known). There were 78 (73\%) who were married or cohabiting and $29(27 \%)$ who were single, divorced, or separated. There were $41(38.3 \%)$ with high school-only education (to age 16), 39 (36.4\%) with further education (to age 18 or below degree level), and 27 $(25.2 \%)$ with higher education (university or degree level). In the UK, at the time of the data collection, 16 years was the

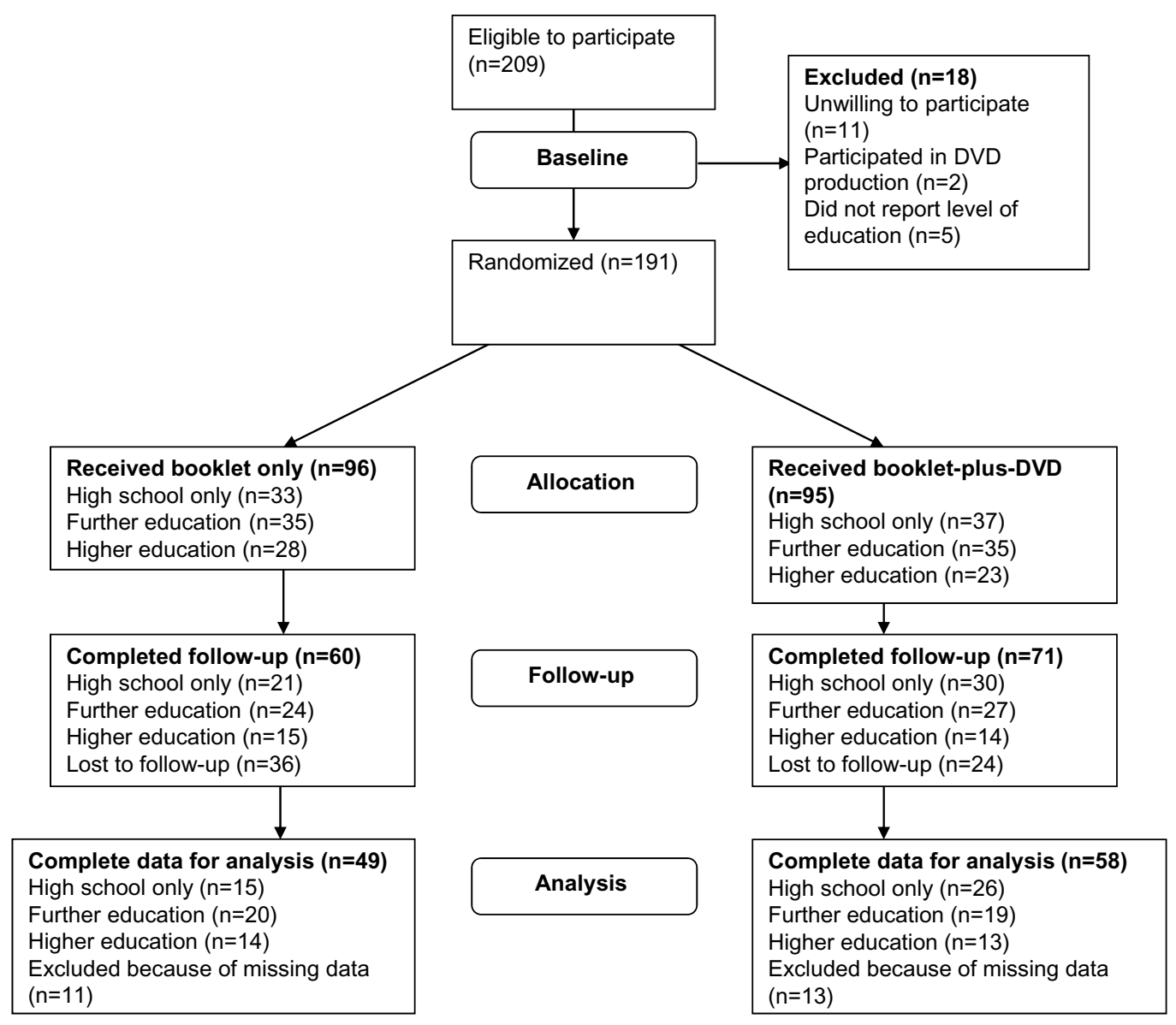

Figure I Study flowchart. 
UK legal minimum school-leaving age, but there is no formal high school graduation in the UK, so the high school-only group corresponds to those in the USA who do not graduate from high school. The further education group corresponds to those in the USA who graduate from high school but do not have a college degree.

\section{Preliminary analyses}

There were no differences between booklet and bookletplus-DVD groups, or between those who did and did not complete follow-up, in terms of proportions with each level of education, marital status, hemophilia type, or hemophilia severity, or in terms of mean age or baseline quality of life or pain scores.

Table 1 shows mean baseline and follow-up quality of life scores. One-way ANOVA with booklet and DVD groups combined showed that education groups differed at baseline in physical quality of life $\left(\mathrm{F}_{(2,101)}=10.724, P<0.001, \eta_{\mathrm{p}}{ }^{2}=0.175\right)$, mental quality of life $\left(\mathrm{F}_{(2,101)}=4.804, P=0.010, \eta_{\mathrm{p}}{ }^{2}=0.087\right)$, and pain $\left(\mathrm{F}_{(2,101)}=10.468, P<0.001, \eta_{\mathrm{p}}^{2}=0.172\right)$. Scheffe post hoc tests showed that those with higher education had greater physical quality of life and less pain than both the other education groups ( $P<0.001$ in each case), and greater mental quality of life than those with high school-only education $(P=0.012)$.

Repeated-measures ANOVA, combining booklet and DVD groups, and also combining education groups, showed a significant increase over time in physical quality of life $\left(\mathrm{F}_{(1,101)}=8.815, P=0.004, \eta_{\mathrm{p}}{ }^{2}=0.080\right)$ and pain $\left(\mathrm{F}_{(1,101)}=4.640, P=0.034, \eta_{\mathrm{p}}^{2}=0.044\right)$ but not mental quality of life $\left(\mathrm{F}_{(1,101)}=0.269, P=0.605, \eta_{\mathrm{p}}^{2}=0.003\right)$.

\section{Main analyses}

ANCOVA to compare quality of life between booklet and booklet-plus-DVD groups at follow-up, with quality of life at baseline included as a covariate, showed that for those with high school-only education, the DVD effect was significant for mental quality of life $\left(\mathrm{F}_{(1,38)}=4.229, P=0.047, \eta_{\mathrm{p}}{ }^{2}=0.100\right)$ but not physical quality of life. For those with further or higher education, there were no significant differences in mental or physical quality of life at follow-up, taking account of baseline quality of life. The secondary analyses showed that there were no significant effects of the DVD on pain subscale scores among any of the education groups.

The DVD effect on mental quality of life among those with high school-only education is illustrated in Figure 2. This shows that for individuals with only high school education, mental quality of life was similar at baseline for those who received only the booklet and those who received the booklet plus DVD, but diverged at follow-up, with higher levels among those who received the DVD. To put the effect of the DVD among those with less education into context, the dotted lines in Figure 2 also show the mean values for individuals with further and higher education (booklet and booklet-plus-DVD groups are combined for simplicity because they did not differ significantly). These show that whereas all of those with only high school education started the trial with lower mental health-related quality of life than the other two groups, those who received the booklet plus DVD achieved a follow-up level of mental quality of life that approximated those with further and higher education.

\section{Supplementary analyses}

Of those who received only the booklet, 67\% (33/49) reported reading it at least once, of whom 49\% (16/33) reported finding it at least "quite helpful". Of those who received the booklet plus DVD, 71\% (41/58) reported reading the booklet at least once, of whom 59\% (24/41) reported finding it at least "quite helpful". There were no significant differences between booklet and booklet-plus-DVD groups in reported use of or perceived helpfulness of the booklet.

Table I Mean (standard deviation) health-related quality of life at baseline and follow-up among groups with different levels of education who received the booklet or booklet-plus-DVD

\begin{tabular}{|c|c|c|c|c|c|c|}
\hline & \multicolumn{3}{|l|}{ Baseline } & \multicolumn{3}{|l|}{ Follow-up } \\
\hline & Booklet only & Booklet-plus-DVD & All & Booklet only & Booklet-plus-DVD & All \\
\hline \multicolumn{7}{|c|}{ High school-only education } \\
\hline Physical quality of life & $27.46(10.86)$ & $28.59(8.97)$ & $28.17(9.58)$ & $29.97(8.32)$ & $29.62(9.63)$ & $29.75(9.07)$ \\
\hline Mental quality of life & 43.31 (I3.29) & 44.11 ( 10.87$)$ & $43.82($ (II.65) & 41.17 (1I.14) & $47.39(10.87)$ & $45.11(11.25)$ \\
\hline \multicolumn{7}{|l|}{ Further education } \\
\hline Physical quality of life & $29.14(11.11)$ & $26.39(8.7 I)$ & $27.80(9.98)$ & $29.76(10.50)$ & 26.82 (II.47) & $28.32(10.94)$ \\
\hline Mental quality of life & $45.57(12.62)$ & $46.85(9.06)$ & $46.19(10.91)$ & $46.29(11.51)$ & $48.07(8.24)$ & $47.16(9.96)$ \\
\hline \multicolumn{7}{|l|}{ Higher education } \\
\hline Physical quality of life & $35.70(10.50)$ & $4 I .2 I(I I .8 I)$ & 38.34 (II.29) & $40.18(9.80)$ & $44.52(\mid 4.21)$ & 42.27 (I2.09) \\
\hline Mental quality of life & $50.90(13.39)$ & $54.02(7.97)$ & 52.40 (I I.02) & $48.37(15.20)$ & $50.66(10.80)$ & $49.48(13.07)$ \\
\hline
\end{tabular}




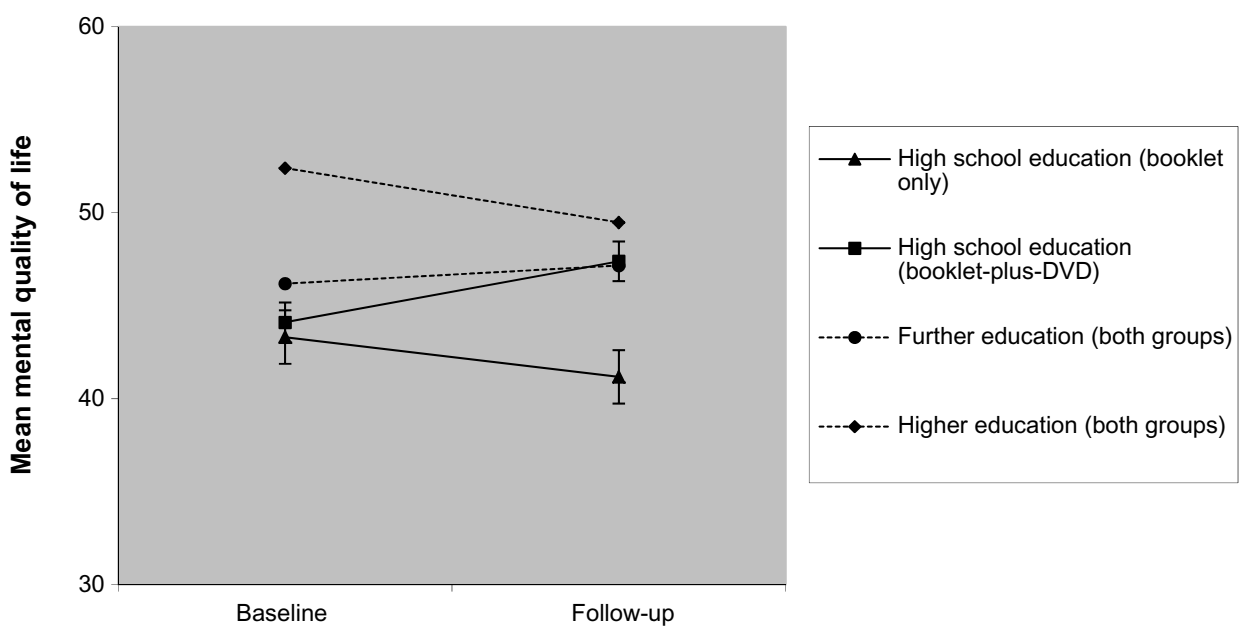

Figure 2 Changes in mental quality of life among participants with different levels of education.

Note: For participants with further and higher education, booklet and booklet-plus-DVD groups are combined for simplicity because they did not differ significantly.

Of those who received the DVD, $39 / 58(67 \%)$ reported watching it at least once, of whom 56\% (22/39) reported finding it at least "quite helpful". There were also no significant differences in reported use or perceived helpfulness between the booklet and DVD among those who received both.

Nor were there any significant differences between education groups in reported use or perceived helpfulness of the booklet or DVD. Of participants with high school-only education, $72 \%$ (28/39) reported reading the booklet at least once, compared with $77 \%$ (30/39) for those with further education and $62 \%(16 / 26)$ of those with higher education. Of those who reported reading the booklet at least once, $46 \%$ $(13 / 28)$ of those with high school-only education reported finding it at least quite helpful, compared with $60 \%(18 / 30)$ of those with further education and 56\% (9/16) of those with higher education.

Of participants with high school-only education who received the DVD, 74\% (17/23) reported watching it at least once, compared with $74 \%$ (14/19) of those with further education and $67 \%(8 / 12)$ of those with higher education. Of those who reported watching the DVD at least once, 53\% (9/17) of those with high school-only education reported finding it at least quite helpful, compared with 57\% (8/14) of those with further education and $63 \%(5 / 8)$ of those with higher education.

Combining education groups to compare those who reported reading the booklet at least once $(n=74)$ with those who did not ( $\mathrm{n}=33)$, those who had read the booklet were older $\left(t_{(102)}=2.68, P=0.009\right)$, and had more pain at both time points (baseline $t_{(102)}=2.63, P=0.010$; follow-up $\left.t_{(102)}=2.70, P=0.008\right)$. Those who read the booklet also had lower physical quality of life at both time points (baseline $t_{(102)}=2.73, P=0.008$; follow-up $t_{(102)}=2.98$, $P=0.004)$, but there were no differences in mental quality of life at either baseline or follow-up.

Among those who received the DVD (again, combining education groups), those who watched the DVD at least once $(n=39)$ did not differ from those who did not ( $n=19)$, in terms of age or baseline and follow-up measures of physical and mental quality of life and pain.

\section{Discussion}

The DVD increased mental health-related quality of life among participants with less education. This group began the trial with lower quality of life than those with more education, and the DVD raised their quality of life toward the level of those with more education, whereas those who received only the booklet did not benefit in the same way.

Several previous studies reported that combined written and video interventions were effective, ${ }^{16-18}$ but this is the first study to our knowledge showing specifically how materials that are mismatched in terms of reading level can be combined to make health education messages more accessible to groups with less education. The DVD was in a narrative style, with messages about the value of pain selfmanagement delivered via personal stories by individual patients. This approach may be particularly well suited for increasing access to health messages among people with less education, for similar narrative-style videos have been shown to have greater impact than purely informational videos in the context of breast cancer health education, ${ }^{13,14}$ especially among people with less education. ${ }^{15}$

This approach could help to make health benefits available to the groups most in need of intervention, 
while preserving the informational value of written material. The potential for this type of application is very wide, for it was estimated that the majority of written patient educational materials currently available were at reading levels that made them inaccessible to the average US adult. ${ }^{6}$ Combined interventions like this could provide benefits for people across levels of education, with written components providing highquality expository information which is made more accessible to people with less education by carefully constructed video components. This approach would be a more effective and cost-effective approach than lowering the reading level of written information or developing video as an alternative to written material.

The DVD had no differential effect on physical healthrelated quality of life or pain, however. It is possible that the narrative style of the DVD, with its emphasis on motivation and attitudes rather than factual presentation of information, gave it more influence on mental than physical health-related quality of life. This is consistent with evidence that mental health-related quality of life in hemophilia is influenced by psychological factors such as pain acceptance, negative thinking, and passive coping, whereas physical healthrelated quality of life is influenced mainly by changes in joint pain. ${ }^{35}$

It is also notable that although the DVD increased mental quality of life among those with less education, this group did not watch the DVD more or rate it as more helpful, compared with the groups with more education. Possibly, the DVD influenced participants in rather subtle ways that they were not immediately aware of, especially if they were more consciously focused on physical health and levels of pain, which were not affected by the DVD. It is also possible that the impact of the DVD was at least partly due to the combined effects of the booklet and DVD, which might not have been captured by the ratings of helpfulness, which were specific to each component.

A potential limitation of the study is that we grouped participants by level of education rather than making individual assessments of literacy or health literacy, and that level of education may overestimate reading level. However, educational level and health literacy are correlated, ${ }^{36,37}$ and one study showed that educational level rather than health literacy was more closely associated with information needs. ${ }^{38}$ Many previous evaluations of health education interventions have examined subgroups based on educational level. ${ }^{8,15,39}$ Literacy and health literacy are complex constructs ${ }^{40}$ and although simplified assessment methods are available, even the simplest require individual assessments, ${ }^{41}$ whereas level of education may be a more straightforward and costeffective criterion for targeting interventions, especially for those designed to be delivered to large groups or sections of the population.

The sample size meant that the study was underpowered compared with the original protocol, which had called for a baseline sample of 200, with three-quarters followed-up. In fact, 191 were randomized, of whom 107 were followed-up and had complete data for analysis. Difficulties with recruitment and data collection in studies of health education interventions often lead to smaller samples and reduced statistical power compared with study protocols, including two of the studies of combined written and filmed health education materials on which the present study builds. ${ }^{17,18}$ In the present study, the final sample was approximately $70 \%$ of the original target, which reduced the statistical power to detect smaller effects, although the size of the effect of the DVD on mental quality of life was small to moderate, as expected. Also, although there was significant attrition from baseline to follow-up, there were not significant differences between those followed-up and those not followed-up.

Our research question focused on the impact of the interventions as received by participants, which was why the sample was restricted to those with good mailing status and the analysis was not on an intent-to-treat basis. However, participants were included in the analysis whether or not they read the booklet and/or viewed the DVD as instructed, so the analysis was on an as-treated rather than per-protocol basis. ${ }^{42}$ This meant that the findings were not based on the subset of participants who used the materials as intended. None of the studies of combined written and filmed health education materials on which the present study built employed intent-to-treat analyses, ${ }^{16-18}$ and we measured nonadherence consistent with recommendations for non-intent-to-treat trials of behavioral interventions. ${ }^{43}$

In fact, the adherence data showed that although most reported reading/watching the booklet/DVD at least once, $31 \%(33 / 107)$ reported not reading the booklet, and 33\% (19/58) reported not watching the DVD. Those who read the booklet were significantly older, and had more pain and lower physical quality of life that those who did not, but there were no significant differences in mental quality of life between those who did and did not read the booklet, and no differences at all between those who did and did not watch the DVD.

That pattern of differences suggests that reading the booklet was influenced mainly by participants' perceived needs, in terms of physical functioning and pain, whereas watching the DVD was not influenced by any measured participant 
characteristics. Perhaps the relative novelty of the DVD meant that those who watched it were just more curious or more interested in video-based materials, or there were other unmeasured factors that made it easier or more convenient for them to view a DVD.

In any case, there was no evidence that adherence to the materials influenced the effect of the DVD on mental quality of life. This raises the question of how people could apparently benefit from materials they did not read or watch. One possibility is that people did not report their use of the DVD accurately, and that better measures of adherence and use are needed to understand how interventions lead to benefits. Another possibility is that merely receiving the DVD prompted changes in attitude or motivation that led to improved mental quality of life, or that receiving the DVD affected how people read and responded to the booklet. More research is needed about how people actually use health education materials like these that are designed to be used very flexibly. The evidence about use of the materials justifies the analytic approach that was adopted in which participants were included in the analysis irrespective of their use of the materials.

However, future research might also include studies of motivational factors that could mediate between interventions like the present one and outcomes like health-related quality of life. In the present study, our focus was on health-related quality of life, because we wished to examine potential benefits in terms of health outcomes, rather than process factors, but if interventions targeting readiness to self-manage are effective, especially for certain groups, then one might expect their impact to be mediated by motivational factors.

This is one of very few studies that reported the readability of the video material. Some studies that combined written and video materials did not report the readability for either, ${ }^{16,17}$ and another reported the readability only of the written materials. ${ }^{18}$ In one of the few studies to report the readability of both, the written and video materials were at the same reading level and were compared directly with one another, ${ }^{11}$ whereas our express intention was to combine materials at different reading levels.

The study also examined health outcomes following the interventions, whereas most such evaluations have focused on short-term changes in knowledge, information recall, or behaviors. ${ }^{20,21}$ In the only other study we know of that also measured health-related quality of life, a combined written and video intervention increased health behaviors, but not health-related quality of life. ${ }^{17}$

The study also extends research on combined, text-plusvideo interventions for people with less education into the context of self-management of chronic pain, where there was previously no such research to our knowledge. One study of acute pain in the emergency department showed no differences between a printed brochure and a video in terms of impact on participants' self-rated pain, and no differential effects by educational level. ${ }^{8}$ Another study of decision making about analgesia for labor and childbirth pain among women in the latter stages of pregnancy showed no differences between a booklet-style and an audio-guided decision aid, and no differential effects by years of education. ${ }^{39}$ The present findings therefore show how health education principles developed in the context of a range of other health conditions can also be applied to promote self-management of chronic joint pain.

\section{Conclusion}

To conclude, the study offers evidence that combining high-reading-level written material with more accessible video material can improve its impact among people with less education. This and further research on how contrasting health education materials can be effectively combined could contribute to future health education initiatives that help to address social and educational health inequalities.

\section{Acknowledgments}

We are very grateful to all the participants in the study; to Tom Bradley and the staff of the Haemophilia Society for undertaking the study mailings; to Ian Kelso, director and producer, and Mark Sydserff, video editor, for production of the DVD; to Bill, Robert, Chris, Jack, and Richard, who featured in the DVD; and to John Morris and Georgina Morrison for their collaboration in the project on which this study builds. We are also grateful to the journal reviewers for their helpful comments on a previous draft.

\section{Disclosure}

This work was supported by the Haemophilia Society UK and the Institute for Health Policy and Research, London Metropolitan University. Baxter and Bayer Healthcare also made small contributions to the costs of the DVD production. The authors report no other conflicts of interest in this work.

\section{References}

1. Luck JV Jr, Silva M, Rodriguez-Merchan EC, Ghalambor N, Zahiri CA, Finn RS. Hemophilic arthropathy. J Am Acad Orthop Surg. 2004;12:234-245.

2. Witkop M, Lambing A, Divine G, Kachalsky E, Rushlow D, Dinnen J. A national study of pain in the bleeding disorders community: a description of haemophilia pain. Haemophilia. 2012;18:e115-e119.

3. Riley RR, Witkop M, Hellman E, Akins S. Assessment and management of pain in haemophilia patients. Haemophilia. 2011;17:839-845. 
4. Elander J. A review of evidence about behavioural and psychological aspects of chronic joint pain among people with haemophilia. Haemophilia. 2014;20:168-175.

5. Cronin M, O'Hanlon S, O'Connor M. Readability level of patient information leaflets for older people. Ir J Med Sci. 2011;180:139-142.

6. Stossel LM, Segar N, Gliatto P, Fallar R, Karani R. Readability of patient education materials available at the point of care. J Gen Intern Med. 2012;27:1165-1170.

7. Aronson ID, Plass JL, Bania TC. Optimizing educational video through comparative trials in clinical environments. Educ Technol Res Dev. 2012;60:469-482.

8. Marco CA, Marco AP, Plewa MC, Buderer N, Bowles J, Lee J. The verbal numeric pain scale: effects of patient education on self-reports of pain. Acad Emerg Med. 2006;13:853-859.

9. Meade CD, McKinney WP, Barnas GP. Educating patients with limited literacy skills: the effectiveness of printed and videotaped materials about colon cancer. Am J Public Health. 1994;84:119-121.

10. Campbell FA, Goldman BD, Boccia ML, Skinner M. The effect of format modifications and reading comprehension on recall of informed consent information by low-income parents: a comparison of print, video, and computer-based presentations. Patient Educ Couns. 2004;53:205-216.

11. Murphy PW, Chesson AL, Walker L, Arnold CL, Chesson LM. Comparing the effectiveness of video and written material for improving knowledge among sleep disorders clinic patients with limited literacy skills. South Med J. 2000;93:297-304.

12. Wilson EA, Park DC, Curtis LM, et al. Media and memory: the efficacy of video and print materials for promoting patient education about asthma. Patient Educ Couns. 2010;80:393-398.

13. Bollinger S, Kreuter MW. Real-time moment-to-moment emotional responses to narrative and informational breast cancer videos in African American women. Health Educ Res. 2012;27:537-543.

14. McQueen A, Kreuter MW, Kalesan B, Alcaraz KI. Understanding narrative effects: the impact of breast cancer survivor stories on message processing, attitudes, and beliefs among African American women. Health Psychol. 2011;30:674-682.

15. Kreuter MW, Holmes K, Alcaraz K, et al. Comparing narrative and informational videos to increase mammography in low-income African American women. Patient Educ Couns. 2010;81 Suppl:S6-S14.

16. France CR, France JL, Wissel ME, Kowalsky JM, BolingerEM, Huckins JL. Enhancing blood donation intentions using multimedia donor education materials. Transfusion. 2011;51:1796-1801.

17. Veroff DR, Sullivan LA, Shoptaw EJ, et al. Improving self-care for heart failure for seniors: the impact of video and written education and decision aids. Popul Health Manag. 2012;15:37-45.

18. Eckman MH, Wise R, Leonard AC, et al. Impact of health literacy on outcomes and effectiveness of an educational intervention in patients with chronic diseases. Patient Educ Couns. 2012;87:143-151.

19. Davis TC, Fredrickson DD, Arnold C, Murphy PW, Herbst M, Bocchini JA. A polio immunization pamphlet with increased appeal and simplified language does not improve comprehension to an acceptable level. Patient Educ Couns. 1998;33:25-37.

20. Wilson EA, Makoul G, Bojarski EA, et al. Comparative analysis of print and multimedia health materials: a review of the literature. Patient Educ Couns. 2012;89:7-14.

21. Pignone M, DeWalt DA, Sheridan S, Berkman N, Lohr KN. Interventions to improve health outcomes for patients with low literacy. A systematic review. J Gen Intern Med. 2005;20:185-192.

Journal of Pain Research

\section{Publish your work in this journal}

The Journal of Pain Research is an international, peer-reviewed, open access, online journal that welcomes laboratory and clinical findings in the fields of pain research and the prevention and management of pain. Original research, reviews, symposium reports, hypothesis formation and commentaries are all considered for publication.

Submit your manuscript here: http://www.dovepress.com/journal-of-pain-research-journal
22. Ferrell BR. The impact of pain on quality of life. A decade of research. Nurs Clin North Am. 1995;30:609-624.

23. Katz N. The impact of pain management on quality of life. J Pain Symptom Manage. 2002;24:S38-S47.

24. Niv D, Kreitler S. Pain and quality of life. Pain Pract. 2001;1:150-161.

25. Tavafian SS, Jamshidi A, Mohammad K, Montazeri A. Low back pain education and short term quality of life: a randomized trial. $B M C$ Musculoskelet Disord. 2007;8:21.

26. Elander J, Robinson G, Morris J. Randomized trial of a DVD intervention to improve readiness to self-manage joint pain. Pain. 2011;152: 2333-2341.

27. Hays RD, Morales LS. The RAND-36 measure of health-related quality of life. Ann Med. 2001;33:350-357.

28. Ware JE, Kosinski M, Keller S. SF-36 Physical and Mental Summary Scales: A user's Manual. Boston: New England Medical Centre; 1994. http://www.worldcat.org/title/sf-36-physical-and-mental-healthsummary-scales-a-users-

29. Fischer K, van Der Bom JG, van den Berg HM. Health-related quality of life as an outcome parameter in haemophilia treatment. Haemophilia. 2003;9 Suppl 1:75-81.

30. Jensen MP, Nielson WR, Kerns RD. Towards the development of a motivational model of pain self-management. J Pain. 2003;4:477-492.

31. Abraham C, Michie S. A taxonomy of behaviour change techniques used in interventions. Health Psychol. 2008;27:379-387.

32. Online-Utility Readability Calculator [webpage on the Internet]. OnlineUtility.org. Available from: http://www.online-utility.org/english/ readability_test_and_improve.jsp. Accessed June 11, 2015.

33. Vickers AJ, Altman DG. Statistics notes: Analysing controlled trials with baseline and follow up measurements. BMJ. 2001;323:1123-1124.

34. Altman, DG, Schultz KF, Moher D, et al. The revised CONSORT statement for reporting randomised trials: explanation and elaboration. Ann Intern Med. 2001;134:663-694.

35. Elander J, Morris J, Robinson G. Pain coping and acceptance as longitudinal predictors of health-related quality of life among people with haemophilia-related chronic joint pain. Eur J Pain. 2013;17:929-938.

36. Paasche-Orlow MK, Parker RM, Gazmararian JA, Nielsen-Bohlman LT, Rudd RR. The prevalence of limited health literacy. J Gen Intern Med. 2005;20:175-184.

37. Pandit AU, Tang JW, Bailey SC, et al. Education, literacy, and health: Mediating effects on hypertension knowledge and control. Patient Educ Couns. 2009;75:381-385.

38. Matsuyama RK, Wilson-Genderson M, Kuhn L, et al. Education level, not health literacy, associated with information needs for patients with cancer. Patient Educ Couns. 2011;85:e229-e236.

39. Raynes-Greenow CH, Roberts CL, Nassar N, Trevena L. Do audio-guided decision aids improve outcomes? A randomized controlled trial of an audioguided decision aid compared with a booklet decision aid for Australian women considering labour analgesia. Health Expect. 2009;12:407-416.

40. Baker DW. The meaning and the measure of health literacy. $J$ Gen Intern Med. 2006;21:878-883.

41. Powers BJ, Trinh JV, Bosworth HB. Can this patient read and understand written health information? JAMA. 2010;304:76-84.

42. Ten Have TR, Normand SL, Marcus SM, Brown CH, Lavori P, Duan N. Intent-to-Treat vs Non-Intent-to-Treat Analyses under Treatment Non-Adherence in Mental Health Randomized Trials. Psychiatr Ann. 2008;38:772-783.

43. Gross D, Fogg L. A critical analysis of the intent-to-treat principle in prevention research. J Prim Prev. 2004;25:475-489.

\section{Dovepress}

The manuscript management system is completely online and includes a very quick and fair peer-review system, which is all easy to use. Visit http://www.dovepress.com/testimonials.php to read real quotes from published authors. 